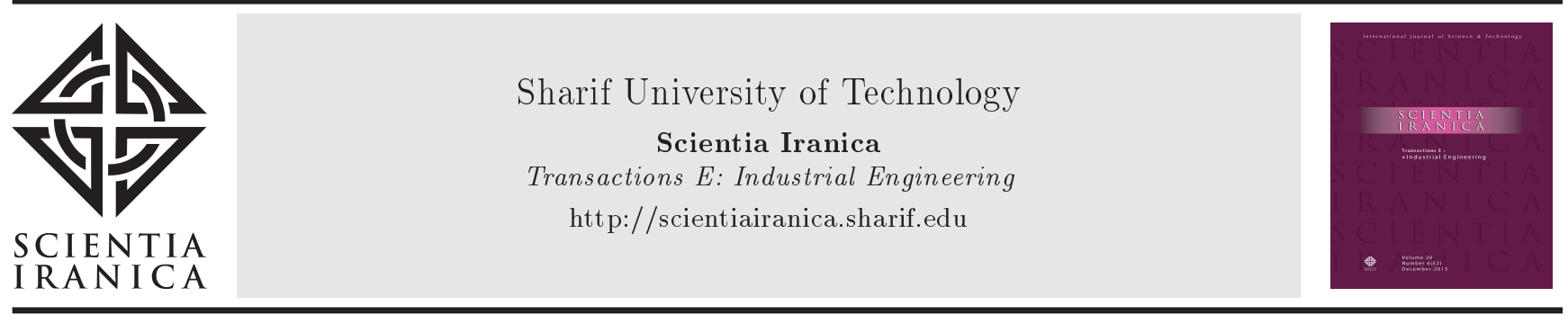

\title{
Cost function and optimal boundaries for a two-level inventory system with information sharing and two identical retailers
}

\author{
A.H. Afshar Sedigh ${ }^{\mathrm{a}}$, R. Haji ${ }^{\mathrm{b}}$, and S.M. Sajadifar ${ }^{\mathrm{c}, *}$ \\ a. Department of Information Science, University of Otago, Dunedin 9054, P. O. Box 56, New Zealand. \\ b. Department of Industrial Engineering, Sharif University of Technology, Tehran, P. O. Box 11365, Iran. \\ c. Department of Industrial Engineering, University of Science and Culture, Tehran, P.O. Box 13145/871, Iran.
}

Received 21 September 2016; received in revised form 22 May 2017; accepted 20 June 2018

\section{KEYWORDS}

Two-echelon inventory system;

Supply chain

management;

Information sharing;

Poisson demand;

Continues review.

\begin{abstract}
In this paper, we consider a two-echelon inventory system with a central warehouse and two identical retailers employing information sharing. Transportation times to each retailer and the warehouse are constant. Retailers face independent Poisson demand and apply continuous review policy, i.e., $(R, Q)$-policy. The warehouse initiates with $m$ batches (of given size $Q$ ) and places an order with an outside supplier when a retailer's inventory position reaches $R+s ; R+s$ is the inventory position considered by the central warehouse and $s$ is a non-negative constant. So far, an approximate cost function as well as exact analysis of system for only one retailer has been proposed. However, the derivation of the exact value of the expected total cost of the system for more than one retailer is still an open question. This paper attempts to meet this challenge and derive the exact cost function for two retailers. To achieve this purpose, we resort to conditional probability to split the problem into two simpler problems; then, we obtain the exact expected total cost of the system.
\end{abstract}

(C) 2019 Sharif University of Technology. All rights reserved.

\section{Introduction}

In an uncertain, complicated, and competitive market, it is vital for a business to improve its flexibility and approachability. To satisfy these needs, companies have concentrated on information technology at an increasingly fast pace. On the other hand, distribution of benefits among members creates a tendency to share their information. If there is a fair distribution of benefits among all members, or all decisions are made

\footnotetext{
*. Corresponding author.

E-mail addresses: amir.afshar@postgrad.otago.ac.nz (A.H. Afshar Sedigh); haji@sharif.edu (R. Haji);

sajadifar@usc.ac.ir (S.M. Sajadifar)
}

doi: $10.24200 /$ sci.2018.20144 by one party, they will use a centralised decisionmaking system. When members are independent and operate as separate parties, these systems are regarded as decentralised supply chain systems.

When there is no information sharing throughout the supply chain, each level aims to optimise its costs. As a result, customer demand variability tends to increase for upstream level parties (vendors, manufacturers, etc.) In the literature, this phenomenon is known as bullwhip effect. Forrester [1] reported this situation for the first time and referred to it as "Demand Amplification." Afterwards, bullwhip effect was shown in games; the most recognised of such games was "Beer Distribution Game," also known as "Beer Game," created by Forrester and others at MIT. Thereafter, electronic versions and web-based versions were proposed by Simchi-Levi et al. [2]. 
Nowadays, we have at our disposal the results of extensive empirical and theoretical studies about the values of information sharing. Lee et al. [3] considered a two-echelon supply chain including one manufacturer and one retailer for periodic review inventory system. Their analysis showed the impact of information sharing on reducing the inventory and cost levels. They also found out that demand process and transportation time had an extensive influence on the advantages of information sharing. Raghunathan and Yeh [4] studied the effect of Continuous Replenishment Program (CRP) on the system proposed by Lee et al.; the presented results showed that for demands with wide variation and small mean, information sharing was more valuable than CRP. Yao and Dresner [5] developed the models proposed by Lee et al. and Raghunathan and Yeh to examine the value of information sharing under CRP and Vendor Managed Inventory (VMI). They concluded that CRP and VMI were more beneficial to the retailer and the manufacturer, respectively. Recently, Dong et al. [6] studied collaborative supply chain management employing an item-level dataset and indicated that the downstream firm gained significant benefits as a result of the decision-transfer inherited from VMI. Cui et al. [7] investigated value of information in a twoechelon supply chain where the decision maker had the permission to change the policy dynamically. Using this approach depicted the efficiency of information sharing as well as reconciliation of theoretical and empirical findings.

In a study, Chen et al. [8] considered eight scenarios with respect to three different types of information, i.e. demand, inventory, and capacity information. These scenarios were designed based on the used information. Their analysis showed that full information sharing had the best performance among other scenarios. Razmi et al. [9] compared traditional information sharing and VMI information sharing systems. They proved that VMI was more advantageous than the traditional approach. Li [10] proposed a flexible method of information sharing in a supply chain where parties could share limited information to other members of supply chain. They performed simulation and quantitative analysis and it was concluded that even limited information sharing would enhance supply chain performance.

Moreover, in [11], a four-echelon supply chain with partial information sharing was simulated. The authors concluded that more information sharing enhanced the system performance; also, increase in collaborating members was an effective factor regardless of their roles. Canella et al. [12] studied the variety of information that could be shared by supply chain parties, i.e. real-time point-of-sale, sales forecasts, and inventory order policies and reports, to improve their performance using a continuous time domain simulation. In [13], Canella and Ciancimino addressed make-to-stock supply chain managing by $(S, R)$ and proposed a novel order-up-to policy for the aforementioned system when parties were coordinated by sharing information. Cannella [14] investigated the enhancement introduced into the supply chain by replacing conventional myopic periodic inventory review with Automatic Pipeline Variable Inventory and Order Based Production Control System (APVIOBPCS) policy when demand was normal.

In more recent studies, Prajogo and Olhager [15] investigated the influence of information sharing in a three-echelon supply chain. Extra demand was backlogged and three levels of information sharing were considered. They concluded that increase in information sharing level resulted in expected cost reduction. Ganesh et al. [16] analysed the value of information sharing in a multi-level supply chain considering various products with multiple levels of substitutability. Their results indicated that if upstream members ignored substitution or demand correlation, they might overestimate the information sharing value. Sabitha et al. [17] studied the value of information sharing in a multi-stage supply chain with one item and non-zero lead time. They found out that the vendor managed inventory and supply-chain-wide information sharing were the same and upstream companies benefited more from this policy. Ali et al. [18] and Babai et al. [19] addressed the value of information sharing in forecasted demand. These two studies indicated that demand process affected the value of information sharing and this value highly diminished in auto-correlated demand. Trapero et al. [20] studied information sharing benefit to forecast improvement. They analysed weekly data of a manufacturer and a major retailer in UK. This study emphasised that information sharing enhanced forecasting accuracy. In a more recent attempt, Cannella et al. [21] simulated a serially linked supply chain consisting of $k$ echelons with decentralised coordination and showed that the collaboration improved service levels of both customers and upstream members of supply chain as well as process efficiency of members.

In the rest of the paper, we concentrate on twolevel inventory systems with stochastic demand and centralised decision-making system. We also narrow down literature review to systems which use continuous review. Li and Wang [22] categorised the proposed methods to handle these systems as follows:

I. Installation stock policies: In these policies, no information about buyers is utilised by the supplier. This property makes them easy to operate, but excludes system performance optimisation. Axsäter [23] proposed a basic model and its 
optimisation for a two-echelon system controlled by a base stock policy using inventory position. Furthermore, Axsäter [24] extended the former model to a batch ordering system for two retailers and suggested an approximation for more than two retailers. Afterwards, Forsberg [25] presented a model and its optimisation for systems which used order up to- $S$ policy with a compound Poisson demand. He showed that demands could be used instead of inventory positions in the basic model proposed by Axsäter. Forsberg [26] also evaluated the exact cost function for a twoechelon system with continuous review policy, Poisson demand, and non-identical batch sizes. Axsäter [27] presented a more generalised model for ordering up to- $S$ policy when demand process was compound Poisson with various ordering size. Further review of stochastic multiproduct systems and evaluating cost using queuing systems were performed by Simchi-Levi and Zhao $[28,29]$.

II. Echelon stock policies: In these systems, a party has information about cumulative inventory position of all related downstream parties. Chen and Zheng [30] evaluated $(R, n Q)$ echelon stock policies in serial inventory systems; they modified an approximation for these systems as well. Chen and Zheng [31] extended this problem to a multistage inventory system with compound Poisson demand and evaluated optimum boundaries, and proposed an algorithm for a near optimal solution. Axsäter and Rosling [32] compared echelon stock and installation stock policies in multi-echelon inventory systems. They concluded that even for the worst scenarios, echelon stock policy was as effective as installation stock policy.

III. Information sharing: In these systems, based on managers' policy, all or part of the information about inventory position, Bill Of Materials (BOM), etc. is shared among members. As it is discussed by Simchi-Levi et al. [2], information sharing can enhance cost savings due to a reduction in the forecast errors which the retailers deal with. The objective of information sharing as a part of information technology is to help supply managers not only to secure transparency and accessibility of information but also to make decisions based on the total supply chain information.

Moinazadeh [33] studied a supply chain including a product, a central warehouse, and some identical retailers under a stationary random demand. In this model, retailers used $(R, Q)$ ordering policy. He recommended an ordering policy for the central warehouse using the real-time information about each retailer's inventory position with the central warehouse placing an order with an external supplier when a retailer's inventory position reached $R+s(0 \leq s \leq Q-1)$. To evaluate the system costs and derive the optimal policy, an approximate model and a heuristic optimization were proposed for this policy.

Haji and Sajadifar [34] derived the exact cost function for the policy proposed by Moinzadeh (2002) considering one retailer. They used Axsäter (1990) installation stock model for one-for-one policy, to evaluate the cost function. Following this study, Sajadifar and Haji [35] derived the optimal boundaries for their proposed model such that their optimisation procedure required less computational effort and was directed towards the optimal solution. In a more recent study, Axsater and Marklund [36] proposed an information sharing policy for non-identical retailers in which demand was a coefficient of a standard batch size and, as previously established, concluded that information sharing lowered the system costs.

In this paper, we evaluate system costs for the policy presented by Moinzadeh (2002) when there are two identical retailers, which has been an open question ever since. A brief schema of the problem is illustrated in Figure 1. The proposed method by Moinzadeh (2002) obtained neither the exact model nor the optimal boundaries of the policy. Moreover, he used a heuristic algorithm that could not guarantee obtaining the optimal solution. To overcome the aforementioned issues, we propose the exact model and optimal boundaries. Therefore, we first propose an illustrative example for this problem to attain better understanding of system complexities, special cases, and different system states. Afterwards, we consider an arbitrary batch $\left(Q_{0}\right)$ and divide the problem into some easier sub-problems to derive the probability function for demands needed to order this batch. Subsequently, we propose upper bound and lower bound of optimum solution and the cost function of the system under study.

The rest of this paper is organised as follows. The problem is defined in Section 2 by proposing its assumptions and notations. In Section 3, an illustrative example is presented for more clarification of complex-

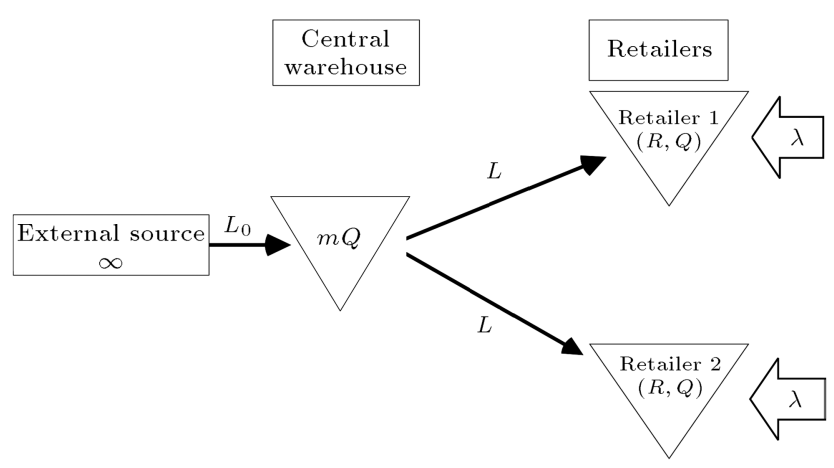

Figure 1. A schema of a two-echelon system with two retailers and a central warehouse. 
ities and difficulties of modelling. Section 4 derives the probability distribution required for cost function calculation. The boundaries of optimal solution are proposed in Section 5. Section 6 represents the final model and its optimisation method. Finally, Section 7 represents the conclusion and some future directions for research.

\section{Problem definition}

In this section, employed notations and assumptions of this paper are represented. We utilise similar notations to those of Axsäter (1990) and Moinzadeh (2002) for ease of researchers familiar with these papers.

\subsection{Notations}

The adopted notations are as follows (similar to [33]):

$L \quad$ Carrying time from the central warehouse to each retailer;

$L_{0} \quad$ Carrying time from the external supplier to the central warehouse;

$\lambda \quad$ Demand intensity at each retailer;

$h \quad$ Holding cost per unit per unit time at each retailer;

$h_{0} \quad$ Holding cost per unit per unit time at the central warehouse;

$\beta \quad$ Shortage cost per unit per unit time at each retailer;

$Q \quad$ Given system's order quantity;

$R \quad$ Reorder point for each one of the retailers;

$m \quad$ Number of initial batches allocated to the central warehouse.

Also, we use the following notations based on [23]:

$\gamma\left(S_{0}\right) \quad$ Average holding cost in the central warehouse per unit when the inventory position at warehouse is $S_{0}$;

$\Pi^{S}\left(S_{0}\right) \quad$ Average holding and shortage cost in a retailer when inventory positions of the central warehouse and retailer are $S_{0}$ and $S$, respectively.

The rest of the notations are as follows (for an example, see Figure 2):

$Q_{0} \quad$ The arbitrary batch chosen for investigation;

$t_{0} \quad$ Ordering time of $Q_{0}$ by the central warehouse;

$t_{1} \quad$ Ordering time of $Q_{0}$ by one of the retailers;

$B \quad$ The retailer which orders $Q_{0}$;

$t^{+} \quad$ A moment after $t$;

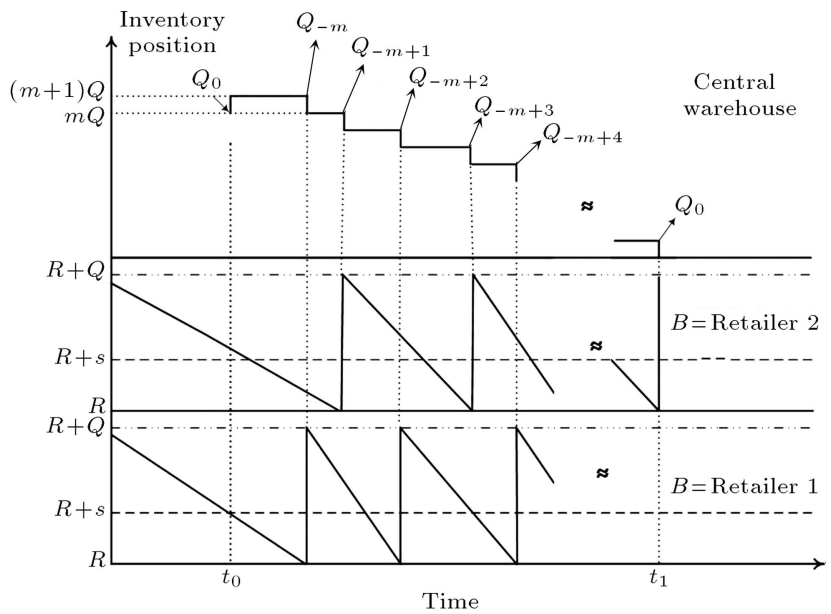

Figure 2. An example of the system.

$\begin{array}{ll}t^{-} & \text {A moment before } t ; \\ k & \text { Customer demand from } t_{0} \text { until } t_{1}^{+} ; \\ j & \text { Arbitrary unit of } Q_{0} ; \\ T C(m, R, s) & \text { Average system cost per unit time. }\end{array}$

\subsection{Assumptions}

In this paper, the following assumptions and policy are considered based on [33]:

1. Carrying times to both the central warehouse and the retailers are constant;

2. Customer demand arrival process at each of the retailers is a Poisson process with a constant and known rate;

3. The shortage will be backlogged;

4. The central warehouse has online information about the inventory position and demand of each one of the retailers;

5. Two retailers have identical demand intensity, holding cost, shortage cost, and carrying time from the central warehouse.

In this model, some of the considered assumptions may be questionable and need justification and discussion. In this part, we discuss the reason behind these assumptions. First, it is worth mentioning that identical retailers is an assumption used in several studies other than Moinzadeh [33]. For instance, Svoronos and Zipkin [37], Deuermeyer and Schwarz [38], Cachon and Fisher [39], Gürbüz et al. [40], Wang et al. [41], and Klastorin et al. [42] addressed identical retailers to simplify mathematical modelling, analysis, and model demonstration. On the other hand, Poisson arrival rate and constant delivery time have extensively been employed in the literature. For instance, Axsäter and Forsberg [23$27,36]$ conducted studies that have considered both constant delivery time and Poisson/compound Poisson 
arrival rates. Moreover, considering shortage backordered is an acceptable assumption. However, there are instances that have considered negligible ordering cost and lost sales. In a study, Haji and Haji [43] proposed a novel policy to handle the aforementioned system. The proposed policy, called $(1, T)$ or one-for-one period ordering policy, aimed to handle such inventory models. In the aforementioned policy, there is no need to share information, since supplier deterministically orders one unit every $T$ units of time. Another assumption of this study is a given batch size and negligible ordering cost. This assumption is widely used in the literature (e.g. [23-27,36-38,43]) and it is acceptable in practice. Some of these instances are considering packaging or shipping requirements as a result of economies of scale in handling, shipping and so forth. Moreover, there are cases that system faces negligible shipping costs or electronic commerce.

Finally, sharing information about demand activities and inventory status in the central warehouse has already been employed in some industries. For instance, Kurt Salmon Associates [44,45] reported employing information technology in the form of Quick Response (QR) and Efficient Customer Response (ECR) in grocery industries. Moreover, Stalk et al. [46] discussed that one of the main reasons for Wal-Mart success was using information system infrastructure that enabled them to exchange information about customer's behaviour in detail. Based on these examples and the former studies, in our research, we assume that information about the on-hand inventory in branches is available and shared with the central warehouse. This assumption is explainable due to the availability of high speed information exchange infrastructures and automated stores. Thus, the following policy is considered in our system.

The central warehouse starts with $m$ initial batches $(m \geq 0)$ and adopts the following ordering policy: When inventory position at a retailer reaches $R+s(0 \leq s \leq Q-1)$, it orders a batch from an external supplier.

\section{An illustrative example}

Let us investigate the system to achieve the central warehouse inventory position. As it can be seen in Figure 3, when a retailer's inventory position reaches $R+s$, inventory position of central warehouse is either $(m+1) Q$ or $(m+2) Q$, considering inventory position of retailers at $t^{-}$(e.g. Figure $4(\mathrm{a})$ and (b)).

Considering $m=3, Q=4$, and $s=2$, Figure 4 indicates independency of the retailer who initiates ordering $Q_{0}$ and the retailer who orders $Q_{0}$. For example, consider the situation that the inventory position of Retailer 1 is $R+2$ at $t_{0}$ (Figure $4(\mathrm{a})$ and (b)); in other words, Retailer 1 initiates ordering $Q_{0}$.

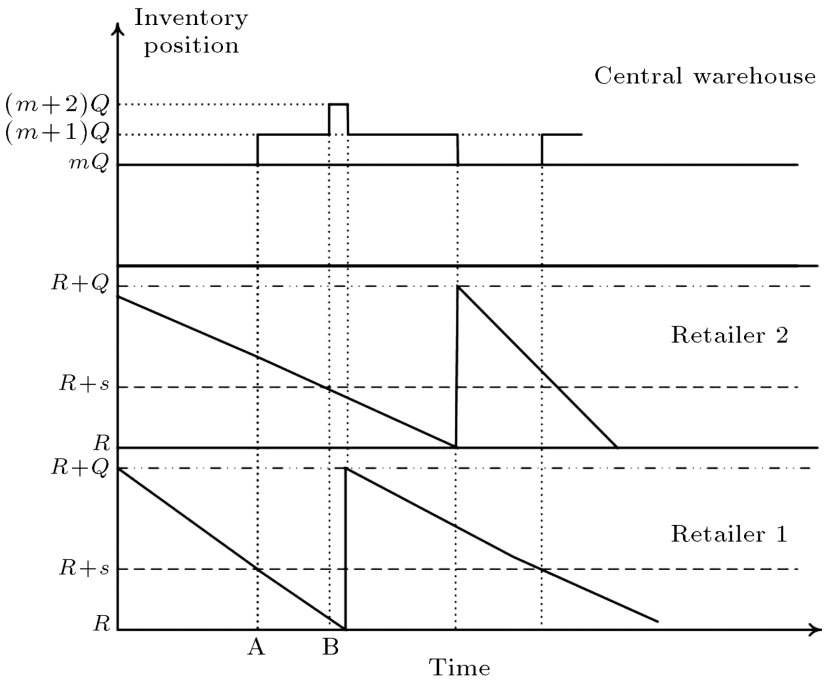

Figure 3. The central warehouse inventory position.

In this situation, based on the customers' demands with each retailer, $Q_{0}$ can be shipped to Retailer 1 (Figure 4(a)) or Retailer 2 (Figure 4(b)). In the rest, for this example, we will consider the case that inventory position of Retailer 1 is equal to $R+s$ at $t_{0}$. Furthermore, Hadley and Whitin [47] showed that at this moment, the inventory position of other retailers, i.e. Retailer 2 , is uniformly distributed between $R+1$ and $R+Q$; thus, the inventory position of the central warehouse is $(m+1) Q$ when $R+s+1 \leq I_{2}\left(t_{0}^{-}\right) \leq R+Q$ with the probability of $(Q-s) / Q$.

Let Retailer 1 order $Q_{0}$; an illustration of this system is available in Figure 5. On the other hand, we know that between $t_{0}$ and $t_{1}^{-}$, three batches are ordered and inventory position of Retailer 1 equals $R+1$ at $t_{1}^{-}$. Knowing that Retailer $i$ can order $b_{i}$ batches, so that $b_{1}+b_{2}=3, b_{i} \geq 0 \forall i$, we want to calculate the probability of ordering these three batches by $l$ demands. Besides, we know that $l=l_{1}+l_{2}$ and $l_{r}$ demands cause the Retailer $r$ to order $b_{r}$ batches from the central warehouse. Considering $l_{r}=b_{r} Q+u_{r}$, we need to obtain the probability that Retailer $r$ receives $l_{r}$ demands from the total of $l$ demands during $t_{1}^{-}-t_{0}$ and the probability that these $l_{r}$ demands initiate $b_{r}$ batches ordering, i.e. $l_{r}=b_{r} Q+u_{r}$.

The probability of distributing $l$ demands between two retailers is $l=l_{1}+l_{2}$ when the demand rates is equal to $\left(\begin{array}{l}l \\ l_{1}\end{array}\right)\left(\frac{1}{2}\right)^{l}$. Moreover, the last demand should occur at Retailer 1. Thus, we eliminate the last demand from the combination. To do so, considering $l^{\prime}=l+1$, $l_{1}^{\prime}=l_{1}+1$, and $l^{\prime}=l_{1}^{\prime}+l_{2}$, the probability is as follows:

$$
\frac{1}{2}\left(\begin{array}{c}
l \\
l_{1}
\end{array}\right)\left(\frac{1}{2}\right)^{l}=\frac{1}{2}\left(\begin{array}{l}
l^{\prime}-1 \\
l_{1}^{\prime}-1
\end{array}\right)\left(\frac{1}{2}\right)^{l^{\prime}-1}=\left(\begin{array}{l}
l^{\prime}-1 \\
l_{1}^{\prime}-1
\end{array}\right)\left(\frac{1}{2}\right)^{l^{\prime}} .
$$

Let $R+k^{\prime}$ show the inventory position of Retailer 2 


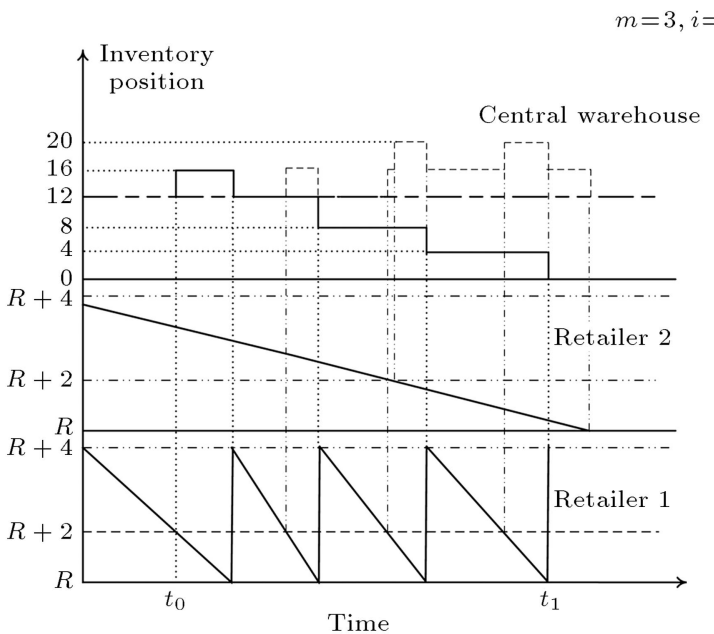

(a)

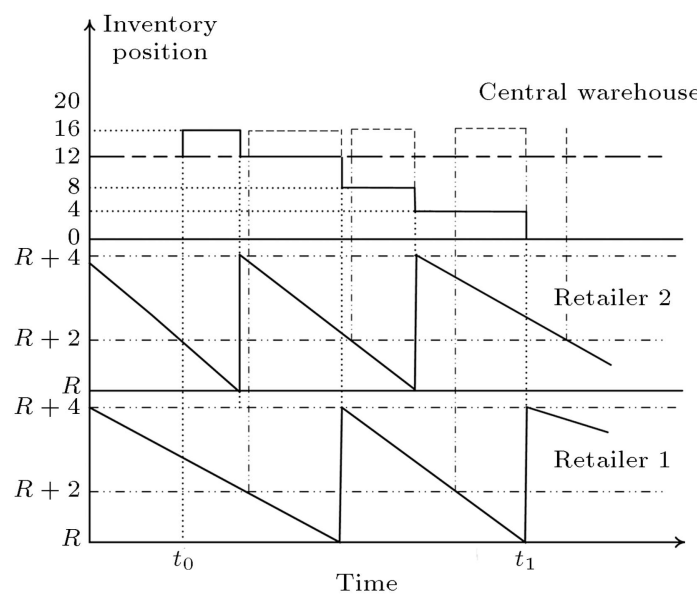

(c)

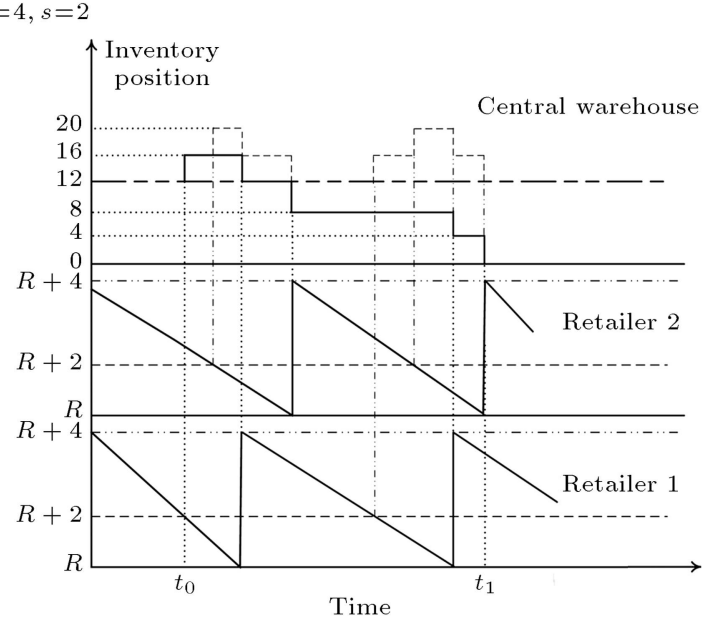

(b)

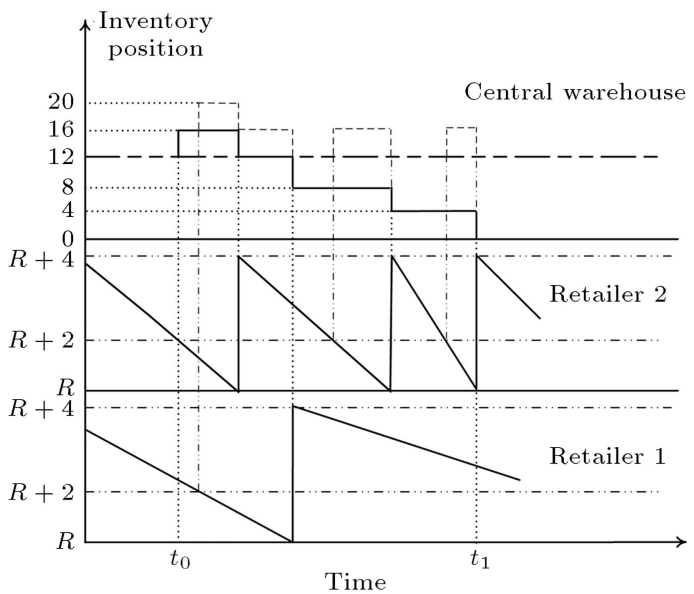

(d)

Figure 4. Different possible combinations of retailers that cause the central warehouse to demand $Q_{0}$ and the retailer that demands $Q_{0}$ from the central warehouse when $I_{w}\left(t_{0}^{-}\right)=m Q$.

at $t_{0}$. For this example, we can calculate the upper and the lower bounds of $l^{\prime}$. To obtain the lower bound, inventory position of Retailer 2 at $t_{1}^{-}$equals $R+Q$; therefore:

$$
l^{\prime} \geq k^{\prime}+4\left(b_{2}-1\right)+4 b_{1}+2 .
$$

On the other hand, we know that $k^{\prime} \geq 3$ and $b_{1}+$ $b_{2}=3$; thus, $l^{\prime} \geq 13$. It is worth mentioning that in Figure 5(a)), the lower bound cannot be met, since $b_{1}=3$ and the lower bound is $4 b_{1}+2=14$, i.e. $l^{\prime}=13$ is not feasible.

To obtain the upper bound, Retailer 2 inventory position should be equal to $R+1$ at $t_{1}^{-}$; therefore, we have:

$$
l^{\prime} \leq k^{\prime}-1+4 b_{2}+4 b_{1}+2 .
$$

On the other hand, we know that $k^{\prime} \leq 4$ and $b_{1}+b_{2}=3$; thus, $l^{\prime} \leq 17$.

Finally, we know that $l^{\prime}=l_{1}^{\prime}+l_{2}$ does not guarantee that all the conditions are held. For example, consider $l_{1}^{\prime}=7$ and $l_{2}=9$; although $13 \leq l^{\prime}=16 \leq 17$, the inventory position of Retailer 1 at $t_{1}^{-}$is equal to $R+4$. On the other hand, consider $l_{1}^{\prime}=14$ and $l_{2}=3$; the inventory position of Retailer 1 at $t_{1}^{-}$is equal to $R+1$. However, with the probability of $1 / 2, k^{\prime}=4$, which leads to a feasible solution. As a result, we need to multiply Eq. (1) by some other probability functions to obtain the probability of demanding 3 batches by these $l^{\prime}$ demands. In other words, we should calculate $P\left(b_{r} Q+u_{r} \rightarrow b_{r}\right), r=1,2$, for this example. Two illustrative computations for $P\left(b_{2} Q+u_{2} \rightarrow b_{2}\right)$ are presented in Figure 6.

\section{Probability distribution of $k$}

Based on Lemmas 1 and 2 proposed in [25], $S_{0}$ and $\mathrm{S}$ are replaced by $k$ and $R+j$, respectively. Hence, the first step for computing the probability distribution is to find the inventory position of the central warehouse and retailers at $t_{0}^{-}$. Based on the warehouse policy, inventory position of one of the retailers is $R+s$ at 


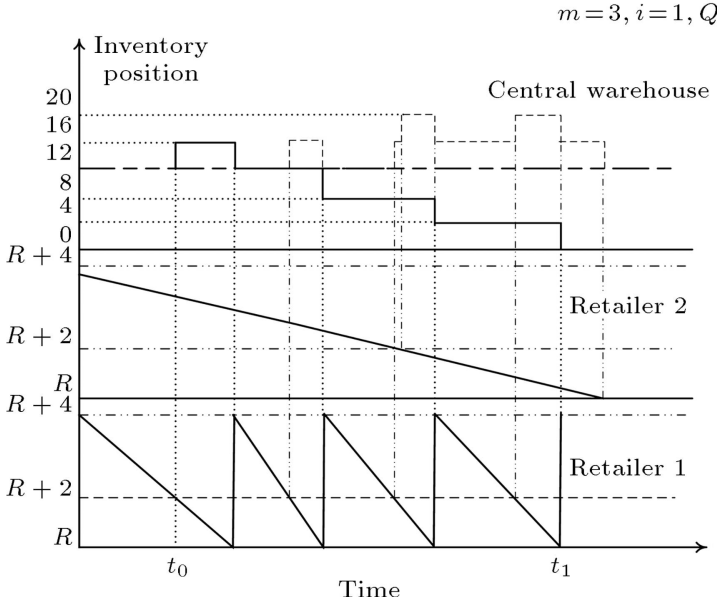

(a) $b_{1}=3, b_{2}=0$

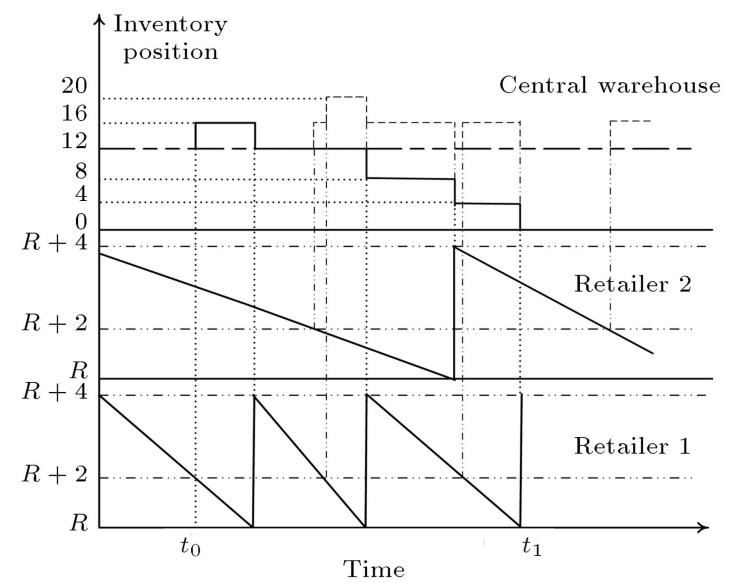

(c) $b_{1}=1, b_{2}=2$

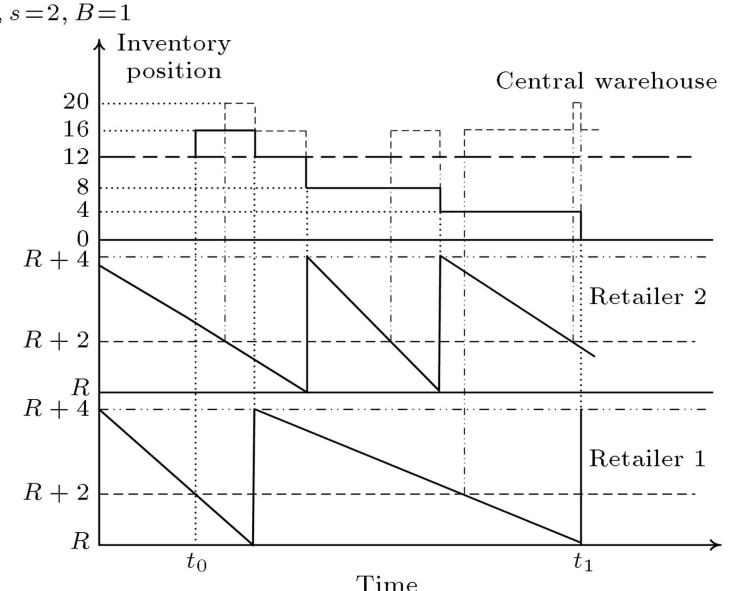

(b) $b_{1}=2, b_{2}=1$

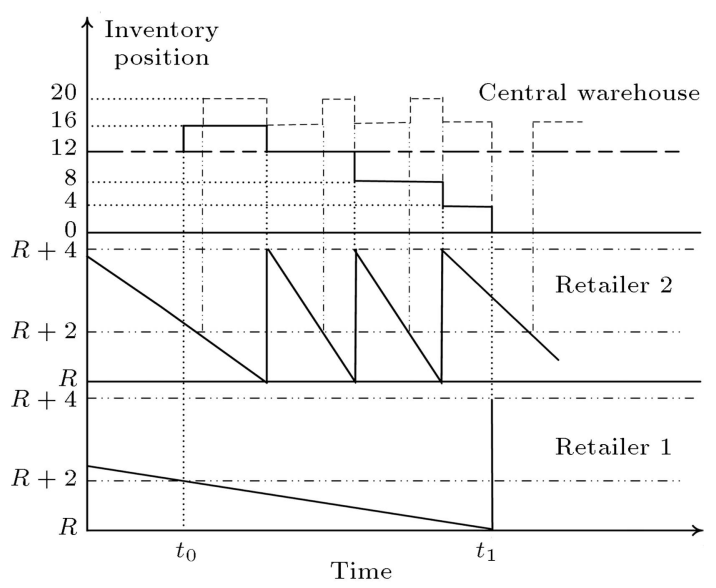

(d) $b_{1}=0, b_{2}=3$

Figure 5. Possible number of batches that Retailers 1 and 2 can order when Retailer 1 causes the central warehouse to order $Q_{0}$ and consume this batch.
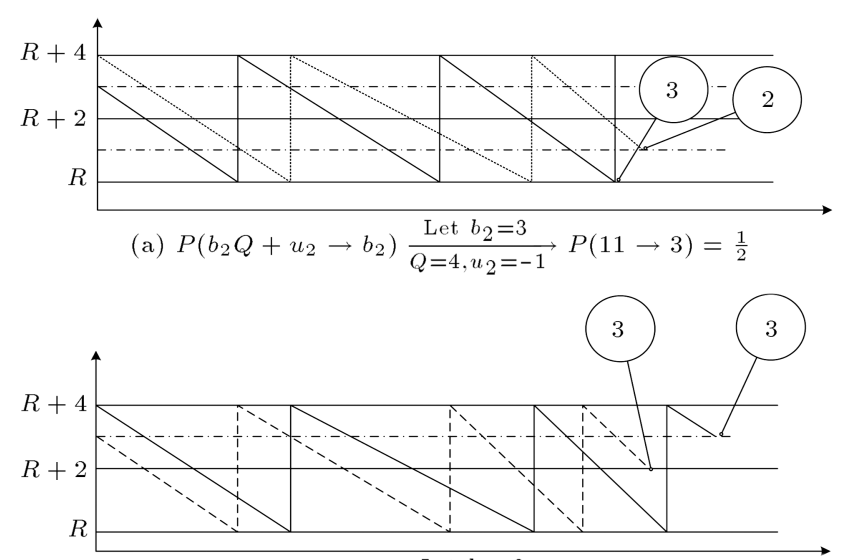

(b) $P\left(b_{2} Q+u_{2} \rightarrow b_{2}\right) \stackrel{\text { Let } b_{2}=3}{Q=4, u_{2}=1} P(13 \rightarrow 3)=1$

Figure 6. Illustrative computations for $P\left(b_{2} Q+u_{2} \rightarrow b_{2}\right)$ when $b_{2}=3$ and $u_{2}=-1$ or $u_{2}=1$.

this moment (without loss of generality, we name it Retailer 1). Furthermore, we know that the inventory position at the central warehouse is related to the last activities in the system. In other words, inventory position at the central warehouse at this moment is a function of $Q, m$, and Retailer 2 inventory position.

\subsection{The central warehouse inventory position at $t_{0}$}

Inventory position for Retailer 2 is uniformly distributed between $R+1$ and $R+Q$. To derive probability distribution of each system state, let $I_{2}\left(t_{0}^{-}\right)$and $I_{c}\left(t_{0}^{-}\right)$ be the inventory positions of Retailer 2 and central warehouse at $t_{0}^{-}$, respectively. As it is shown in Figure 3 , at $\mathrm{A}$ and $\mathrm{B}, I_{c}\left(t_{0}^{-}\right)$is $m$ and $m+1$, respectively:

$$
\begin{aligned}
& P(i=1, s)=P\left(I_{c}\left(t_{0}^{-}\right)=m\right) \\
& \quad=P\left(R+s+1 \leq I_{2}\left(t_{0}^{-}\right) \leq R+Q\right)=\frac{Q-s}{Q} \\
& P(i=2, s)=P\left(I_{c}\left(t_{0}^{-}\right)=m+1\right) \\
& \quad=P\left(R+1 \leq I_{2}\left(t_{0}^{-}\right) \leq R+s\right)=\frac{s}{Q}
\end{aligned}
$$

To calculate the probability of ordering $b$ batches 
by Retailer $r$, let us introduce the following notations (note that $s$ is one of the system parameters):

$\mu_{r, l, B, s}^{i} \quad$ This random variable demonstrates the number of system batches ordered by Retailer $r$ with $l$ demands, when there are $m+i$ batches at the central warehouse;

$\eta_{2, l, B, s}^{i} \quad$ This random variable demonstrates the number of system batches ordered by the retailers by $l$ demands from these retailers, when there are $m+i$ batches at the central warehouse.

Note that, to compute $\mu_{r, l, B, s}^{i}$, the last customer demand is disregarded to ensure that it is the last one among all customer demands. This demand with probability of $\frac{1}{2}$ is assigned to Retailer $B$. Therefore, for computing $\eta_{2, l, B, s}^{i}$, we have:

$$
\begin{aligned}
\operatorname{Pr} & \left(\eta_{2, l, B, s}^{i}=b\right)=\sum_{k=0}^{l-1} \sum_{b^{\prime}=0}^{b-1}\left(\begin{array}{l}
l \\
k
\end{array}\right)\left(\frac{1}{2}\right)^{l} \\
& \times \operatorname{Pr}\left(\mu_{1, l-k-1, B, s}^{i}=b-b^{\prime}-1\right) \\
& \times \operatorname{Pr}\left(\mu_{2, k, B, s}^{i}=b^{\prime}\right) .
\end{aligned}
$$

Since inventory position of each retailer is renewed for every $Q$ customer demands, evaluating system demands will be easier using $b Q+u$. Hence, we can rewrite $\operatorname{Pr}\left(\mu_{r, l, B, s}^{i}=b\right)$, considering $l=b Q+u$, as $\operatorname{Pr}\left(\mu_{r, b Q+u, B, s}^{i}=b\right)$. The value of this probability depends on $s, u, i$, and $r$; $i$ shows that there is $m+i$ system batches at the central warehouse and $s$ is known by system policy.

\subsection{Investigating the system for $s>0$}

We calculate $\operatorname{Pr}\left(\mu_{r, b Q+u, B, s}^{i}=b\right)$ for $r=1$ and $r=2$, separately.

\subsubsection{Probability of ordering $b$ batches by Retailer 1}

To compute this probability, we first investigate which retailer orders $Q_{0}$. If $B=1$, then at $t_{1}^{-}$, the inventory position of this retailer is $R+1$. Therefore, we have:

$$
\begin{gathered}
\operatorname{Pr}\left(\mu_{1, b Q+u, 1, s>0}^{i}=b\right)=1 \quad \text { if } \quad B=1, \\
u=s-1, \quad b \geq 0, \quad \forall i .
\end{gathered}
$$

On the other hand, if $B \neq 1$, then for this retailer, we know that $I_{r}\left(t_{0}\right)=R+s$; we can conclude that $b^{\prime} Q+s \leq D<b^{\prime} Q+Q+s$ surely causes $b^{\prime}+1$ batch orderings. Defining $b=b^{\prime}+1$ and $u=D-b Q$, we have:

$$
\begin{aligned}
& \operatorname{Pr}\left(\mu_{1, b Q+u, 1, s>0}^{i}=b\right)=1 \\
& \quad r=1, \quad B=2, \quad s>0, \quad s-Q \leq u<0, \quad b \geq 1,
\end{aligned}
$$

or:

$$
r=1, \quad B=2, \quad s>0, \quad 0 \leq u, \quad b \geq 0 .
$$

4.2.2. Probability of ordering $b$ batches by Retailer 2 Let us consider Retailer 2 when $R+1 \leq I_{2}\left(t_{0}^{-}\right) \leq R+s$ and $B=2$. Therefore, we know that $I_{2}\left(t_{1}^{-}\right)=R+1$. Thus:

$$
\begin{gathered}
\operatorname{Pr}\left(\mu_{2, b Q+u, 2, s>0}^{2}=b\right)=\frac{1}{s}, \\
0 \leq u \leq s-1, \quad b \geq 0 .
\end{gathered}
$$

Now, consider the situation that $B=1$; we know that $R+1 \leq I_{2}\left(t_{0}^{-}\right) \leq R+s$ and $R+1 \leq I_{2}\left(t_{1}^{-}\right) \leq R+Q$. Therefore, to order $b$ batches by $b Q+u$ demands, the following equation should be held:

$$
\begin{aligned}
b Q & +u+I_{2}\left(t_{0}^{-}\right)=b Q+I_{2}\left(t_{1}^{-}\right) \Rightarrow u \\
& =I_{2}\left(t_{1}^{-}\right)-I_{2}\left(t_{0}^{-}\right) \Rightarrow 1-Q \leq u \leq s-1 .
\end{aligned}
$$

To calculate the probability, two states are considered:

(I) $0 \leq u \leq s-1$ or

(II) $-Q+1 \leq u \leq-1$.

In state (I), $u$ should not exceed $I_{r}\left(t_{0}\right)-R$; this occurs if $I_{r}\left(t_{0}\right)>R+u$. On the other hand, $I_{r}\left(t_{0}\right)$ is uniformly distributed over $\{R+1, \cdots, R+s\}$. Therefore, the probability is as follows:

$$
\begin{aligned}
& \operatorname{Pr}\left(\mu_{2, b Q+u, 1, s>0}^{2}=b\right)=\frac{s-u}{s}, \\
& 0 \leq u \leq s, \quad b \geq 0 .
\end{aligned}
$$

State (II) is categorised in two subdivisions:

(II-a) $s-Q<u \leq-1$ : In this subdivision, customer demands are equal to $u+Q$ and the interval can be modified as $s<u+Q \leq Q$. These customer demands surely trigger a retailer order, because they exceed $I_{r}\left(t_{0}\right)$. Thus, the related probability is:

$$
\begin{gathered}
\operatorname{Pr}\left(\mu_{2, b Q+u, 1, s>0}^{2}=b\right)=1 \\
s-Q<u<0, \quad b \geq 1 .
\end{gathered}
$$

(II-b) $-Q+1<u \leq s-Q$ : As $0<u+Q \leq s$, if these customer demands are equal to or greater than $I_{r}\left(t_{0}\right)-R$, then the retailer orders a new batch. Thus, probability of $u+Q \geq I_{r}\left(t_{0}\right)$ is as follows:

$$
\begin{gathered}
\operatorname{Pr}\left(\mu_{r, b Q+u, B, s}^{i}=b\right)=\frac{Q+u}{s}, \\
-Q<u \leq s-Q, \quad b>0 .
\end{gathered}
$$


The following probabilities can be computed with the same justification:

$$
\begin{aligned}
& \operatorname{Pr}\left(\mu_{2, b Q+u, 1, s>0}^{1}=b\right) \\
& \quad= \begin{cases}1+\frac{u}{Q-s} & s-Q<u \leq 0, \quad b \geq 1 \\
1 & 0 \leq u \leq s-1, \quad b \geq 0 \\
\frac{Q-u}{Q-s} & s<u \leq Q, \quad b \geq 0\end{cases} \\
& \operatorname{Pr}\left(\mu_{2, b Q+u, 2, s>0}^{1}=b\right)=\frac{1}{Q-s}, \\
& s \leq u<Q, \quad b \geq 0 .
\end{aligned}
$$

\subsection{Investigating the system for $s=0$}

Here, $\operatorname{Pr}\left(\mu_{r, b Q+u, B, s}^{i}=b\right)$ is acceptable when $B \geq 1$ and the last demand must be considered in order to avoid negative customer demand in the special case of $B=1$ and $b=0$. It is also obvious that at $t_{0}$, one batch is ordered by Retailer 1 and the central warehouse, simultaneously. Thus, $I_{c}\left(t_{0}^{+}\right)=m+i$ and $I_{1}\left(t_{0}^{+}\right)=Q$.

Therefore, the probabilities of demanding $b$ batches by Retailer $r$ are defined as follow:

$$
\begin{gathered}
\operatorname{Pr}\left(\mu_{2, b Q+u, 2,0}^{1}=b\right)=\frac{1}{Q}, \\
0 \leq u<Q, \quad b \geq 0, \\
\operatorname{Pr}\left(\mu_{2, b Q+u, 2,0}^{1}=b\right) \\
= \begin{cases}\frac{Q+u}{Q} & -Q \leq u<0, b \geq 1 \\
\frac{Q-u}{Q} & 0 \leq u<Q, b \geq 0\end{cases} \\
\operatorname{Pr}\left(\mu_{2, b Q+u, 2,0}^{1}=b\right)=1, \\
B=2, \quad 0 \leq u<Q, \quad b \geq 0 \\
B=1, \quad u=Q-1, \quad b \geq 0 .
\end{gathered}
$$

Furthermore, for $m=0$, the $B$ batch is ordered at $t_{0}^{-}$ by Retailer 1 and central warehouse, simultaneously. Thus, surely there is no need for any demand other than the mentioned one; therefore, $k=0$.

\section{Boundaries of customers' demand}

Let us introduce the following notations:

$l b(i, s, m) \quad$ Lower bound of customer demand for a known $s$, when system starts with $m$ initial batches and $I_{c}\left(t_{0}^{+}\right)=m+i$;

$u b(i, s, m) \quad$ Upper bound of customer demand for a known $s$, when system starts with $m$ initial batches and $I_{c}\left(t_{0}^{+}\right)=m+i$.

\subsection{Lower bounds}

For $s>0$, we know:

$$
\left\{\begin{array}{cc}
\min \left\{I_{2}\left(t_{0}\right)\right\}=R+1 & I_{c}\left(t_{0}^{+}\right)=m+2 \\
I_{1}\left(t_{0}\right)=R+s & \\
\min \left\{I_{2}\left(t_{0}\right)\right\}=R+s+1 & I_{c}\left(t_{0}^{+}\right)=m+1
\end{array}\right.
$$

Consider $I_{c}\left(t_{0}^{+}\right)=m+2$; after meeting $R$, every retailer should have at least $Q$ demands to order a new batch. As a result, two batches can be ordered by $s+1$ demands from each retailer and the remaining $m$ batches are ordered by $m Q$ demands. Furthermore, we disregard the last customer demand; therefore, we have:

$$
l b(2, s>0, m)=s+1+m Q .
$$

Now, we investigate $I_{c}\left(t_{0}^{+}\right)=1$ and $m=0$. At least $s$ demands from Retailer 1 can cause ordering a new batch. Thus:

$$
l b(1, s>0,0)=s .
$$

On the other hand, for $m>0$, with a justification as before, the lower bound will be:

$$
l b(1, s>0, m>0)=2 s+1+(m-1) Q .
$$

We can summarise these bounds as:

$$
\begin{aligned}
l b & (i, s>0, m) \\
\quad & = \begin{cases}s & m+i=1 \\
(3-i) s+1+(m+i-2) Q & m+i \geq 2\end{cases}
\end{aligned}
$$

When $s=0$, we know that $I_{c}\left(t_{0}^{+}\right)=m$ and we have the following inventory positions for retailers:

$$
\left\{\begin{array}{l}
I_{1}\left(t_{0}^{+}\right)=R+Q \\
\min \left\{I_{2}\left(t_{0}^{+}\right)\right\}=R+1
\end{array}\right.
$$

Hence, at most one batch can be ordered by one customer demand and the remaining batches, if any, are ordered by $Q$ customer demands. Therefore, generally, the lower bounds are:

$$
\begin{aligned}
& l b(i, s, m) \\
& \quad=\left\{\begin{array}{lll}
0 & m=0, \quad s=0 \\
(m-1) Q+1 & m \geq 1, & s=0 \\
s & m+i=1, & s>0 \\
(3-i) s+(m+i-2) Q+1 & \text { Otherwise }
\end{array}\right.
\end{aligned}
$$




\subsection{Upper bounds}

When $s>0$, we know that:

$\max \left\{I_{r}\left(t_{0}\right)\right\}:$

$$
\begin{cases}\max \left\{I_{2}\left(t_{0}\right)\right\}=R+s & I_{c}\left(t_{0}^{+}\right)=m+2 \\ I_{1}\left(t_{0}\right)=R+s & \\ \max \left\{I_{2}\left(t_{0}\right)\right\}=R+Q & I_{c}\left(t_{0}^{+}\right)=m+1\end{cases}
$$

Consider $I_{c}\left(t_{0}^{+}\right)=m+i$; the most possible demand occurs when one of the retailers has $b Q+\max \left\{I_{r}\left(t_{0}\right)\right\}-$ $R$ demands and the other one has $b^{\prime} Q+\max \left\{I_{r^{\prime}}\left(t_{0}\right)\right\}-$ $(R+1)$, where $b^{\prime}+b=m+i-1$. Thus, for $I_{c}\left(t_{0}^{+}\right)=$ $m+1$, we have:

$$
\begin{aligned}
u b(1, s>0, m) & =s+Q-1+m Q \Rightarrow \\
& =s-1+(m+1) Q .
\end{aligned}
$$

And for $I_{c}\left(t_{0}^{+}\right)=m+2$, we have:

$$
u b(2, s>0, m)=2 s-1+(m+1) Q .
$$

Consequently, for $s>0$, we obtain Eq. (25):

$$
u b(i, s>0, m)=i s-1+(m+1) Q .
$$

For $s=0$ and $m>0$, we know that:

$$
\max \left\{I_{r}\left(t_{0}^{+}\right)\right\}:\left\{\begin{array}{l}
I_{1}\left(t_{0}^{+}\right)=R+Q \\
\max \left\{I_{2}\left(t_{0}^{+}\right)\right\}=R+Q
\end{array}\right.
$$

Thus, Eq. (27) is calculated as follows:

$$
u b(1,0, m>0)=(m+1) Q-1 .
$$

Finally, the only difference between $s=0$ and $s>0$ is in the batch quantities. As mentioned before, for $s=0$, we consider $m$ batches instead of $m+1$. Therefore, we have:

$$
u b(i, s, m)= \begin{cases}i \times s-1+(m+1) Q & s>0 \\ (m+1) Q-1 & s=0, \quad m>0\end{cases}
$$

\section{The final model and its optimisation}

In this study, an arbitrary unit of $Q_{0}$ is considered, which can be the $j$ th unit of $Q_{0}$ with probability of $1 / Q$. Furthermore, this unit should wait for $R+j$ additional demands from $t_{1}^{+}$. Thus, we have Eq. (29) as shown in Box I. This method is an extension of the method presented by Axsäter (1990) [23] (see Appendix A).

Let us introduce the following notations based on [23]:

$G^{s}(t) \quad$ Cumulative distribution function of Erlang $(N \lambda, S)$, which is calculated as follows:

$$
\sum_{k=S}^{\infty} \frac{(\lambda t)^{k}}{k !} e^{-\lambda t}=1-\sum_{k=0}^{S-1} \frac{(\lambda t)^{k}}{k !} e^{-\lambda t} .
$$

$\pi^{S}(t) \quad$ Conditional expected cost of $\Pi^{S}\left(S_{0}\right)$ knowing that $\Delta=t$, which is independent of $S_{0}$;

$M_{s} \quad$ The upper bound for the number of initial batches in the central warehouse $(m)$ for a given $s$ with respect to the acceptable error;

$m_{s}^{l} \quad$ The lower bound for $m$ for a given $s$;

$m_{s}^{u} \quad$ The upper bound for $m$ for a given $s$.

For large values of $S_{0}, \Pi^{S}\left(S_{0}\right)$ can be approximated by $\pi^{S}(0)$; therefore, for an acceptable error $(\varepsilon), G^{M}\left(L_{0}\right)<\varepsilon$. On the other hand, for a given $s, l b(1, s, m)=\min \{l b(i, s, m)\}_{\forall i}$. Thus:

$T C(m, s, R)$

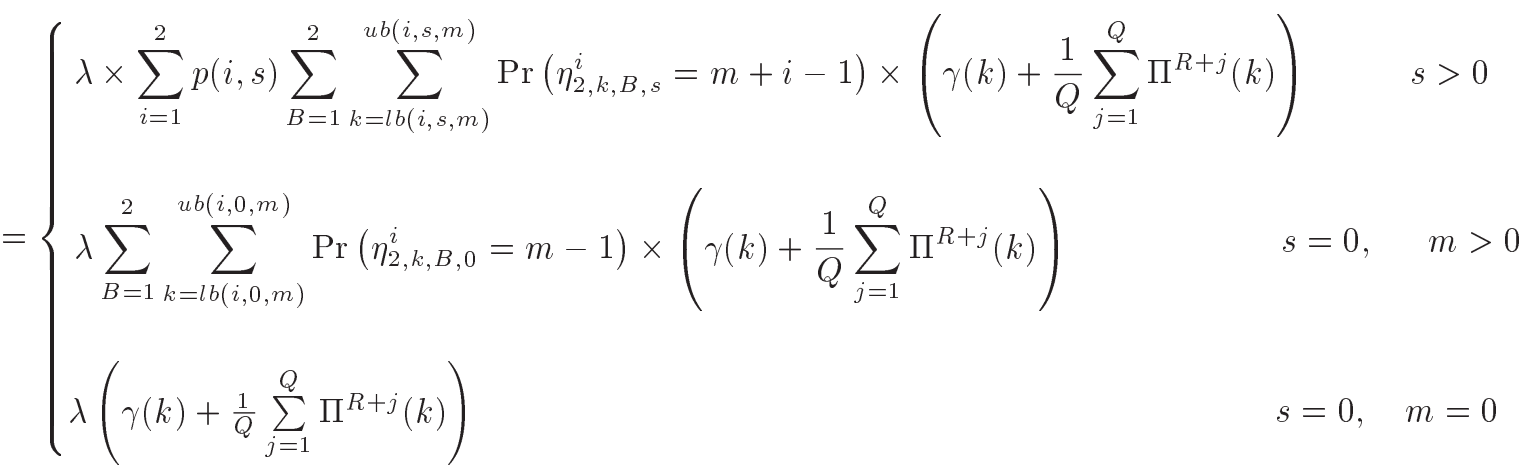




$$
M_{s}= \begin{cases}0 & M=0, \quad s=0 \\ \left\lceil\frac{M-1}{Q}+1\right\rceil & M>0, \quad s=0 \\ \left\lceil\frac{M-s}{s+1}\right\rceil & M \leq 2 s+1, \quad s>0 \\ \left\lceil\frac{M-2 s-1}{Q}+1\right\rceil & M>2 s+1, \quad s>0\end{cases}
$$

where, $\lceil x\rceil$ is ceiling, i.e. the smallest integer greater than or equal to $x$. Furthermore, let the upper bound and the lower bound of $R$ be $R_{l}$ and $R_{u}$. Due to the convexity of $\Pi^{S}\left(S_{0}\right)$ with respect to $S$ for a known $S_{0}, R_{l}$ and $R_{u}$ can be calculated by minimization of $\frac{1}{Q} \sum_{j=1}^{Q} \pi^{R+j}\left(L_{0}\right)$ and $\frac{1}{Q} \sum_{j=1}^{Q} \pi^{R+j}(0)$, respectively. Finally, the lower bounds for $R_{l}$ and $R_{u}$ are $-Q$ and $R_{l}$. Knowing $R_{l}$ and $R_{u}$, for a given $s, m_{s}^{u}$ and $m_{s}^{l}$ can be calculated as follows:

$$
\left\{\begin{array}{l}
m_{s}^{l}=\min _{0 \leq m \leq M_{s}}\left\{T C\left(m, s, R_{u}\right)\right\} \\
m_{s}^{u}=\min _{m_{s}^{l} \leq m \leq M_{s}}\left\{T C\left(m, s, R_{l}\right)\right\}
\end{array}\right.
$$

The following lemma leads to less computational efforts:

Lemma: To compute $R_{l}^{i}(0)$ and $R_{u}^{i}(0)$, it is enough to compare $\pi^{R+1}(\cdot)$ with $\pi^{R+Q+1}(\cdot)$ using the convexity attribute.

Proof: Optimal $R_{l}^{i}(0)$ and $R_{u}^{i}(0)$ are obtained by comparing $\frac{1}{Q} \sum_{j=1}^{Q} \pi_{i}^{R^{i}+j}(\cdot)$ with $\frac{1}{Q} \sum_{j=1}^{Q} \pi_{i}^{R^{i}+1+j}(\cdot)$. Consider $A=\sum_{j=2}^{Q} \pi^{R+j}(\cdot)$; therefore:

$$
\frac{1}{Q} \sum_{j=1}^{Q} \pi^{R+j}(\cdot)=\frac{1}{Q}\left(\pi^{R+1}(\cdot)+A\right)
$$

and:

$$
\frac{1}{Q} \sum_{j=1}^{Q} \pi^{R+1+j}(\cdot)=\frac{1}{Q}\left(\pi^{R+Q+1}(\cdot)+A\right) .
$$

Since $A$ and $\frac{1}{Q}$ are common parameters, they can be ignored.

\section{Conclusions}

In this paper, we modelled a two-level inventory system with two identical retailers that benefited from an information sharing policy. We proposed the exact model and a procedure to find optimal solutions to this problem. It is worth mentioning that both of them have been open questions up to now. We first proposed an illustrative example to identify system states and provide better understanding of system behaviour in different situations. To resolve the complexity of the proposed problem, we divided the main problem into some easier sub-problems; then, we modelled the whole system by merging the sub-problems. Furthermore, we considered an arbitrary batch and derived probability distribution for the number of required demands to order this batch. Next, we proposed the boundaries of optimum variables and the final model for the proposed system.

By modelling and proposing optimisation procedure for an easy to implement information sharing policy, this study can be used as a baseline for a deeper knowledge of how this policy works. It is worth mentioning that the simplicity of this policy, i.e. using limited parameters and information about inventory status and demand, makes it easy to implement in real-world scenarios in a way that managers have a sense of the inventory management scheme and the way it operates. Moreover, the shared information in this system is an acceptable range of information that is shared in distributer companies, i.e. inventory status and demand. Furthermore, the policy under study takes advantage of fast developing information technology in forms of information transfer infrastructures, radio-frequency identification (RFID), and computational speed to propose an easy to implement inventory control scheme without any need to share crucial information or face data overload. The simplicity of this policy may be claimed to be a double-edged sword; however, considering the continuously growing arguments about information sharing disadvantages, the tendency to limit shared information in this model can be its positive aspect.

As future subjects of study, the exact model for more than two retailers for the proposed policy is still an open question. In addition, extending this problem to non-identical retailers and making the policy more flexible are attractive problems to study. Moreover, considering different policies with limited dynamicity are other attractive subjects of study.

\section{References}

1. Forrester, J.W. "Industrial dynamics: a major breakthrough for decision makers", Harvard Business Review, 36(4), pp. 37-66 (1958).

2. Simchi-Levi, D., Kaminsky, P., and Simchi-Levi, E., Designing and Managing the Supply Chain: Concepts, Strategies, and Case Studies, The McGraw-Hill/Irwin Series in Operations and Decision Sciences, McGrawHill, Illinois (2003).

3. Lee, H.L., So, K.C., and Tang, C.S. "The value 
of information sharing in a two-level supply chain", Management Science, 46(5), pp. 626-643 (2000).

4. Raghunathan, S. and Yeh, A.B. "Beyond EDI: impact of continuous replenishment program (CRP) between a manufacturer and its retailers", Information Systems Research, 12(4), pp. 406-419 (2001).

5. Yao, Y. and Dresner, M. "The inventory value of information sharing, continuous replenishment, and vendor-managed inventory", Transportation Research Part E: Logistics and Transportation Review, 44(3), pp. 361-378 (2008).

6. Dong, Y., Dresner, M., and Yao, Y. "Beyond information sharing: An empirical analysis of vendor-managed inventory", Production and Operations Management, 23(5), pp. 817-828 (2014).

7. Cui, R., Allon, G., Bassamboo, A., and Van Mieghem, J.A. "Information sharing in supply chains: An empirical and theoretical valuation", Management Science, 61(11), pp. 2803-2824 (2015).

8. Chen, M.-C., Yang, T., and Yen, C.-T. "Investigating the value of information sharing in multi-echelon supply chains", Quality \& Quantity, 41(3), pp. 497-511 (2007).

9. Razmi, J., Rad, R.H., and Sangari, M.S. "Developing a two-echelon mathematical model for a vendormanaged inventory (VMI) system", The International Journal of Advanced Manufacturing Technology, 48(58), pp. 773-783 (2010).

10. Li, C. "Controlling the bullwhip effect in a supply chain system with constrained information flows", Applied Mathematical Modelling, 37(4), pp. 1897-1909 (2013).

11. Cannella, S., Dominguez, R., Framinan, J.M., and Bruccoleri, M. "Insights on partial information sharing in supply chain dynamics", International Conference on Industrial Engineering and Systems Management (IESM), Seville, Spain, pp. 344-350 (2015).

12. Cannella, S., Framinan, J.M., and Barbosa-Póvoa, A. "An IT-enabled supply chain model: a simulation study", International Journal of Systems Science, 45(11), pp. 2327-2341 (2014).

13. Cannella, S., and Ciancimino, E. "Up-to-date supply chain management: The coordinated $(\mathrm{S}, \mathrm{R})$ orderup-to", In Advanced Manufacturing and Sustainable Logistics, Dangelmaier, W., Blecken, A., Delius, A., and S. Klöpfer, Eds., pp. 175-185, Springer, Berlin, Germany (2010).

14. Cannella, S. "Order-up-to policies in information exchange supply chains", Applied Mathematical Modelling, 38(23), pp. 5553-5561 (2014).
15. Prajogo, D. and Olhager, J. "Supply chain integration and performance: The effects of long-term relationships, information technology and sharing, and logistics integration", International Journal of Production Economics, 135(1), pp. 514-522 (2012).

16. Ganesh, M., Raghunathan, S., and Rajendran, C. "The value of information sharing in a multi-product, multi-level supply chain: Impact of product substitution, demand correlation, and partial information sharing", Decision Support Systems, 58, pp. 79-94 (2014).

17. Sabitha, D., Rajendran, C., Kalpakam, S., and Ziegler, $H$. "The value of information sharing in a serial supply chain with AR (1) demand and non-zero replenishment lead times", European Journal of Operational Research, 255(3), pp. 758-777 (2016).

18. Ali, M.M., Boylan, J.E., and Syntetos, A.A. "Forecast errors and inventory performance under forecast information sharing", International Journal of Forecasting, 28(4), pp. 830-841 (2012).

19. Babai, M.Z., Boylan, J.E., Syntetos, A.A., and Ali, M.M. "Reduction of the value of information sharing as demand becomes strongly auto-correlated", International Journal of Production Economics, 181, pp. 130-135 (2016).

20. Trapero, J.R., Kourentzes, N., and Fildes, R. "Impact of information exchange on supplier forecasting performance", Omega, 40(6), pp. 738-747 (2012).

21. Cannella, S., López-Campos, M., Dominguez, R., Ashayeri, J., and Miranda, P.A. "A simulation model of a coordinated decentralized supply chain", International Transactions in Operational Research, 22(4), pp. 735-756 (2015).

22. Li, X. and Wang, Q. "Coordination mechanisms of supply chain systems", European Journal of Operational Research, 179(1), pp. 1-16 (2007).

23. Axsäter, S. "Simple solution procedures for a class of two-echelon inventory problems", Operations Research, 38(1), pp. 64-69 (1990).

24. Axsäter, S. "Exact and approximate evaluation of batch-ordering policies for two-level inventory systems", Operations Research, 41(4), pp. 777-785 (1993).

25. Forsberg, R. "Optimization of order-up-to-S policies for two-level inventory systems with compound Poisson demand", European Journal of Operational Research, 81(1), pp. 143-153 (1995).

26. Forsberg, R. "Exact evaluation of (R, Q)-policies for two-level inventory systems with Poisson demand", European Journal of Operational Research, 96(1), pp. 130-138 (1997).

27. Axsäter, S. "Exact analysis of continuous review (R,Q) policies in two-echelon inventory systems with compound Poisson demand", Operations Research, 48(5), pp. 686-696 (2000). 
28. Simchi-Levi, D. and Zhao, Y. "Three generic methods for evaluating stochastic multi-echelon inventory systems", Working Paper, Massachusetts Institute of Technology, Cambridge (2007).

29. Simchi-Levi, D. and Zhao, Y. "Performance evaluation of stochastic multi-echelon inventory systems: a survey", Advances in Operations Research, 2012, Article ID: 126254,34 pages (2012). https://doi.org/10.1155/2012/126254

30. Chen, F. and Zheng, Y.-S. "Evaluating echelon stock $(\mathrm{R}, \mathrm{nQ})$ policies in serial production/inventory systems with stochastic demand", Management Science, 40(10), pp. 1262-1275 (1994).

31. Chen, F. and Zheng, Y.-S. "Near-optimal echelonstock (R, nQ) policies in multistage serial systems", Operations Research, 46(4), pp. 592-602 (1998).

32. Axsäter, S. and Rosling, K. "Notes: Installation vs. echelon stock policies for multilevel inventory control", Management Science, 39(10), pp. 1274-1280 (1993).

33. Moinzadeh, K. "A multi-echelon inventory system with information exchange", Management Science, 48(3), pp. $414-426$ (2002).

34. Haji, R. and Sajadifar, S.M. "Deriving the exact cost function for a two-level inventory system with information sharing", Journal of Industrial and Systems Engineering, 2(1), pp. 41-50 (2008).

35. Sajadifar, S.M. and Haji, R. "Optimal solution for a two-level inventory system with information exchange leading to a more computationally efficient search", Applied Mathematics and Computation, 189(2), pp. 1341-1349 (2007).

36. Axsäter, S. and Marklund, J. "Optimal position-based warehouse ordering in divergent two-echelon inventory systems", Operations Research, 56(4), pp. 976-991 (2008).

37. Svoronos, A. and Zipkin, P. "Estimating the performance of multi-level inventory systems", Operations Research, 36(1), pp. 57-72 (1988).

38. Deuermeyer, B.L., and Schwarz, L.B., A Model for the Analysis of System Service Level in WarehouseRetailer Distribution Systems: the Identical Retailer Case, Purdue University (1981).

39. Cachon, G.P. and Fisher, M. "Supply chain inventory management and the value of shared information", Management Science, 46(8), pp. 1032-1048 (2000).

40. Gürbüz, M.Ç., Moinzadeh, K., and Zhou, Y.P. "Coordinated replenishment strategies in inventory/distribution systems", Management Science, 53(2), pp. 293-307 (2007).

41. Wang, Q., Chay, Y., and Wu, Z. "A simple coordination strategy for a decentralized supply chain", Working Paper, Nanyang Business School, Nanyang Technological University, Singapore (2006).
42. Klastorin, T.D., Moinzadeh, K., and Son, J. "Coordinating orders in supply chains through price discounts", IIE Transactions, 34(8), pp. 679-689 (2002).

43. Haji, R. and Haji, A. "One-for-one period policy and its optimal solution", Journal of Industrial and Systems Engineering, 1(2), pp. 200-217 (2007).

44. Kurt Salmon Associates, Inc., Efficient Consumer Response: Enhancing Consumer Value in the Grocery Industry, Research Department Food Marketing Institute, Washington, Dc (1993).

45. Kurt Salmon Associates, Inc., Quick Response: Meeting Customer Need, Kurt Salmon Associates, Atlanta, GA (1997).

46. Stalk, G., Evans, P., and Shulman, L.E. "Competing on capabilities: The new rules of corporate strategy", Harvard Business Review, 70(2), pp. 57-69 (1991).

47. Hadley, G. and Whitin, T.M., Analysis of Inventory Systems, Prentice Hall, Englewood, Cliffs, NJ (1963).

\section{Appendix A}

In this part, a summary of [23] cost functions is proposed.

$\lambda_{0}$ is demand intensity at the central warehouse $\left(\sum_{i=1}^{N} \lambda_{i}\right)$. Note that in our paper, $\lambda_{0}$ is equal to $N \lambda$.

The average holding cost in the central warehouse $\gamma\left(S_{0}\right)$ is:

$$
\gamma\left(S_{0}\right)=e^{-\lambda_{0} L_{0}} \frac{h_{0}}{\lambda_{0}} \cdot \sum_{k=0}^{S_{0}-1} \frac{\left(S_{0}-k\right)}{k !}\left(\lambda_{0} L_{0}\right)^{k}, \quad S_{0}>0 .
$$

Or equally:

$$
\begin{aligned}
\gamma\left(S_{0}\right)= & \frac{h_{0} S_{0}}{\lambda_{0}} \cdot\left(1-G_{0}^{S_{0}+1}\left(L_{0}\right)\right) \\
& -h_{0} L_{0}\left(1-G_{0}^{S_{0}}\left(L_{0}\right)\right), \quad S_{0}>0 .
\end{aligned}
$$

For $S_{0} \leq 0$, we have:

$$
\gamma\left(S_{0}\right)=0, \quad S_{0} \leq 0 .
$$

The unit cost at retailer $i$ for a given delay equal to $t$ at the warehouse is:

$$
\begin{aligned}
\pi_{i}^{S_{i}}(t)= & e^{-\lambda_{i}\left(L_{i}+t\right)} \frac{h_{i}+\beta_{i}}{\lambda_{i}} \cdot \sum_{k=0}^{S_{i}-1} \frac{\left(S_{i}-k\right)}{k !} \lambda_{i}^{k}\left(L_{i}+t\right)^{k} \\
& +\beta_{i}\left(L_{i}+t-\frac{S_{i}}{\lambda_{i}}\right)
\end{aligned}
$$

For $S_{i} \leq 0$, average holding and shortage cost in retailer $i, \Pi_{i}^{S_{i}}\left(S_{0}\right)$, is:

$$
\begin{aligned}
\Pi_{i}^{S_{i}}\left(S_{0}\right)= & G_{0}^{S_{0}}\left(L_{0}\right) \beta_{i} L_{0}-G_{0}^{S_{0}+1}\left(L_{0}\right) \beta_{i} \frac{S_{0}}{L_{0}} \\
& +\beta_{i}\left(L_{i}-\frac{S_{i}}{L_{i}}\right), \quad S_{i} \leq 0, \quad S_{0}>0
\end{aligned}
$$

and: 


$$
\Pi_{i}^{S_{i}}\left(S_{0}\right)=\beta_{i}\left(L_{i}+L_{0}-\frac{S_{i}}{L_{i}}-\frac{S_{0}}{L_{0}}\right)
$$

$$
S_{i} \leq 0, \quad S_{0} \leq 0
$$

For $S_{i}>0$, it is possible to obtain the following recursive equation:

$$
\begin{array}{r}
\Pi_{i}^{S_{i}}\left(S_{0}-1\right)=\frac{\lambda_{i}}{\lambda_{0}} \Pi_{i}^{S_{i}-1}\left(S_{0}\right)+\frac{\lambda_{0}-\lambda_{i}}{\lambda_{0}} \Pi_{i}^{S_{i}}\left(S_{0}\right) \\
+\frac{\lambda_{i}}{\lambda_{0}}\left(1-G_{0}^{S_{0}}\left(L_{0}\right)\right)\left(\pi_{i}^{S_{i}}(0)-\pi_{i}^{S_{i}-1}(0)\right),
\end{array}
$$

$$
S_{0}>0 \text {. }
$$

For $S_{0} \leq 0$, we have:

$$
\Pi_{i}^{S_{i}}\left(S_{0}-1\right)=\frac{\lambda_{i}}{\lambda_{0}} \Pi_{i}^{S_{i}-1}\left(S_{0}\right)+\frac{\lambda_{0}-\lambda_{i}}{\lambda_{0}} \Pi_{i}^{S_{i}}\left(S_{0}\right),
$$

$$
S_{0} \leq 0 .
$$

$\Pi_{i}^{0}\left(S_{0}\right)$ can be obtained by Eqs. (A.5) and (A.6).

Finally, for sufficiently large values of $S_{0}$, the delay at warehouse is equal to 0 ; thus, we have:

$$
\Pi_{i}^{S_{i}}\left(S_{0}\right) \approx \pi_{i}^{S_{i}}(0) .
$$

This approximation is asymptotically exact. To determine an initial large value of $S_{0}$, let the probability that the warehouse can deliver without a delay be smaller than $\varepsilon$ :

$$
G_{0}^{\overline{S_{0}}}\left(L_{0}\right)<\varepsilon,
$$

where $\varepsilon$ is a small positive number.

\section{Biographies}

Amir Hosein Afshar Sedigh is currently a PhD candidatein Information Science at University of Otago, Dunedin, New Zealand. He received a BSc degree in Industrial Engineering from Qazvin Islamic Azad University, Qazvin, Iran. Thereafter, he received his MSc degree in Industrial Engineering from Sharif University of Technology, Tehran, Iran. His research interests include supply chain management, inventory control, and queuing theory.

Rasoul Haji is currently a Professor of Industrial Engineering at Sharif University of Technology in Tehran, Iran. He received a BSc degree from University of Tehran in Chemical Engineering in 1964. In 1967, he received his MSc degree and then, in 1970, his PhD degree both in Industrial Engineering from the University of California-Berkeley. He is recognized as a co-founder of a fundamental and important relation in queuing theory known as "Distributional Little's Law". His research interests include inventory control, stochastic processes, and queuing theory.

Seyed Mehdi Sajadifar is currently an Assistant Professor of Industrial Engineering at University of Science and Culture in Tehran, Iran. He received a BSc degree from Sharif University of Technology in Industrial Engineering in 1995. In 1997, he received his MSc degree and then, in 2008, after experiencing 5 years of academic teaching, his $\mathrm{PhD}$ degree in Industrial Engineering both from Sharif University of Technology. His research interests include inventory management, supply chain management, and operations research. 